EMPLOYING A VIRTUAL REALITY TOOL TO EXPLICATE TACIT KNOWLEDGE OF MACHINE OPERATORS

*A. Vasenev, T. Hartmann, and A.G. Dorée

University of Twente

5 Drienerlolaan

Enschede, The Netherlands $7522 \mathrm{NB}$

(*Corresponding author: a.vasenev@utwente.nl) 


\title{
EMPLOYING A VIRTUAL REALITY TOOL TO EXPLICATE TACIT KNOWLEDGE OF MACHINE OPERATORS
}

\begin{abstract}
The quality and durability of asphalted roads strongly depends on the final step in the road construction process; the profiling and compaction of the fresh spread asphalt. During compaction machine operators continuously make decisions on how to proceed with the compaction accounting for projectspecific aspects as: ambient conditions, road geometry, roller type, asphalt mixture characteristics and behavior of other machines.

In discussions over quality improvement in road construction it is often suggested to robotize rollers. To operate such robots would of course require operational strategies and routines. The reality of this moment is that these operational strategies and routines are not documented. To identify the existing best working practices and, ultimately, to proceed with developing automated robotized compactors, the knowledge of machine operators should at first be explored and described in a formalized way. Unfortunately operators have difficulty verbalizing their expertise. Observation of behavior, as machine movement patterns, could be helpful to extract the operational strategies, but such observations are time and labor consuming. To overcome this burden we developed a Virtual Reality (VR) environment. In this VR environment operators perform compaction virtually, and their operational choices are traced and analysed.

This paper describes this VR development and explains how it helps in following machine operators and extracting their (tacit) professional knowledge. The road geometry and the working conditions are visually represented; the operators show - rather than explain - how they would proceed with the compaction process in the given conditions. Movements of virtual machines are documented, analysed, visualised and discussed. This VR approach is expected to contribute to learning, to more formal description of operational strategies; stepping stones towards compaction algorithms for roller robotisation.
\end{abstract}

\section{KEYWORDS}

Visualization, tacit knowledge, road compaction, rolling pattern, virtual reality

\section{INTRODUCTION}

Road building and Hot Mix Asphalt (HMA) paving in particular is machine intensive. Experienced operators operate dedicated machines as pavers and several types of compacters. Advances in HMA mixtures, contractual restrictions, and machine-, sensor- and information technologies challenge the current practice. Debates over road quality and quality control often bring forward the question if and when compaction could be robotised. To do so in the long run we must first understand the process compaction and get insight into the operational strategies of the operators.

The compaction process is crucial to the quality of the road; if the obtained density is not sufficient, the pavement will distort and lose its original shape under heavy loads (Lavin, 2003). The process of compaction highly depends on human decisions. It requires coordinated movements of rollers to homogeneously compact the asphalt mat to the required degree before the asphalt mixture cools down, making further compaction pernicious. 
Project specific conditions, including asphalt mixture properties, environmental conditions, and layer thickness (Asphalt Institute, 1989), require roller operators to choose a compaction strategy, for example which part of the road to compact first, what distance to keep from the paver, roller speed, many short of fewer long strokes, turning points, etc. These choices are based on the operators' adopted common practices, tacit knowledge, and his personal judgment (Huerne ter, Miller \& Dorée, 2007). The selected strategy is operationalized by operators' decisions where to go at every moment in time, making the roller continuously move and reverse its direction. Segments of roller's travelling in one direction constitute a rolling pattern, which characterizes how the machine moved to compact the asphalt mat.

To handle various asphalt mixtures, machine operators need to know appropriate compaction strategies for various conditions. Best practices exist in the form of operators' tacit knowledge. This knowledge is normally acquired by experience, learning by doing and peer advice, as the knowledge can hardly be verbalized due to its tacit and complicated nature (Miller, 2010).

To foster sharing best practices there is a need for an approach to explicate operators' knowledge, for example by examining rolling patterns. In this paper we employ a virtual reality environment that supports operators in constructing moving patterns under specific conditions. The movements of a virtual roller within this environment can then be documented and quantitatively analysed.

The next sections of this paper are organized as follows: first, specifics of the asphalt compaction process are described; second, the visualizations usage in construction is shortly overviewed; last, we describe the implemented VR environment to explicate tacit knowledge of machine operators. The paper concludes with reflections on value of the VR environment as a stepping-stone towards more atomization in the compaction.

\section{SPECIFICS OF THE ASPHALT COMPACTION PROCESS}

Compaction begins immediately after an asphalt mixture is spread by a paving machine (NCDoTMaT, 2012) and continues until the asphalt mat is compacted to a certain density. The mat continuously cools down (Vasenev, Hartmann \& Dorée, 2012) and should be uniformly compacted while the in-asphalt temperature is within particular limits. If operators attempt to compact while the temperature is outside of the range, they can damage the asphalt mat. For instance, if rollers compact the hot mixture too close to the paver, the material can be displaced rather than compacted, and if the mat is already cooled down below a certain temperature, further rolling can damage it. In this way, the roller operator should consider the asphalt temperature and compact at some distance from the paver.

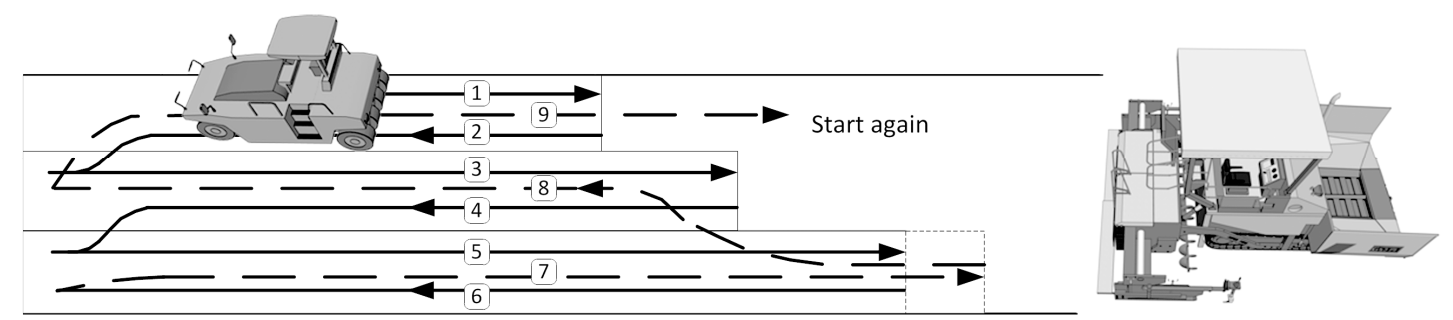

Figure 1. An exemplary rolling pattern to uniformly compact the asphalt mat that requires three passes

Often more than one compaction strategy can be suitable for any road geometry. For example, compaction of a straight road can be performed in different rolling patterns (Tserng et. al., 1996). The decision on which pattern to use or, in other words, how to roll, depends on project-specific requirements and the operators' preferable working methods. The operators should consider, for example, whether the asphalt mat was laid next to a cold or a warm asphalted layer and how many times every roller should travel over the road surface. In addition, distances between different rollers and their distances from the paver should be chosen carefully based on the cooling rate of the asphalt mat. For illustrative purposes a 
relatively straightforward pattern that results in three roller passes over every patch of asphalt is represented in Figure 1. This rolling pattern provides quick coverage of the mat, most lane changes happen on the fully compacted area of the mat, and it smoothly feeds into the first pass of the next pattern repetition (NAPA, 1996). With introduction of more complicated road geometries, the pattern should be modified. If the road includes curves, conjunctions, or roundabouts, rolling patterns normally become more entangled. For instance, for rolling curves machine operators usually choose from two different strategies on how to compact: they can compact from the inner side to the outer side throughout the turn or, alternatively, compact in tangential direction (Floss, 2001). Similarly, using different rollers within the compaction fleet can influence the rolling patterns.

Choosing a suitable compaction strategy for particular conditions implies the need to know best practices and working methods adopted during the professional development process of compactor operators. Consequently, sharing operators' knowledge among professionals is a matter of the utmost importance.

Though the scope and the parameters of the compaction process are known and well described, little is known about the way the operators given a specific situation translate the task to operational strategies. Their behaviour seems to be largely emergent from tacit knowledge and built up experience.

\section{TOWARDS UNDERSTANDING AND IMPROVING COMPACTION STRATEGIES}

Tacit knowledge is connected to personal experience and cannot be easily passed on to others (Krogh, Ichijo \& Nonaka, 2000). Correspondingly, tacit knowledge is normally transferred by direct observations or experimentation (Nonaka \& Takeuchi, 1995) that requires physical proximity. For roller operators, this process of knowledge transfer takes place during compaction operations, but this transfer is limited only to people at the construction site. One way to share best practices to a larger audience is explicating operators' knowledge by documenting and analysing machine movements.

Retrospective visualizations proved to be an effective means to analyze construction processes and to speed up process learning (Miller, Hartmann \& Dorée, 2011). However, collecting and analysing documented real-world construction projects is a time consuming process due to several reasons. First, data collection requires travelling to and from the construction site, installing and de-mounting navigation sensors, and processing the sensors reading. Second, the data from the real projects can highly depend on the particular road geometry and specific weather conditions. The latter can obscure possibilities of comparing different projects. In such complexity one could follow the route of gathering data of many projects and many potential relevant parameters. After that statistical analysis could then reveal patterns. This is a time and cost consuming pathway. Design and conduct of controlled experiments is a strategy to reduce the variance in parameters and the complexity of the analysis. For paving and compaction that would still require substantial financial investments. Currently virtual reality and gaming are the next step in controlled experimentation. This VR and gaming route is selected for explication of the operators' tacit reasoning and rules for operation. Although the VR and gaming are used in construction research, such a VR environment specifically HMA compaction was not yet available and needed to be developed.

\section{Using Virtual Reality Environments in Construction}

Virtual reality stands for a "mosaic of technologies that support the creation of synthetic, highly interactive three dimensional (3D) spatial environments that represent real or non-real situations" (Mikropoulos \& Natsis, 2011). These technologies can serve as an additional means to visually express knowledge in addition to expressing it by the print and oral language media (Greenfield, 2009).

In the construction industry, VR environments are mainly used for simulation and visualization purposes of, for example, earthwork, and underground facilities projects (Kang et. al., 2010). Virtual reality environments were utilized to visualize and optimize process flows at construction sites (Cheng \& Teizer, 2012), facilitate the visual validation and simulation of project progress (Retik \& Shapira, 1999), or 
support educating students or practitioners (Chavada \& Dawood, 2010). Specialized VR environments, such as SimPave and AsphaltOpen, also simulate and visualize asphalt paving operations. For instance, the SimPave pavement construction simulation system was designed to provide opportunities to experiment with the effect of resource and equipment allocation on the schedule, budget, and quality of a pavement construction task (Turkiyyah \& Northwest, 2007). The visualization system AsphaltOpen was developed to support understanding of asphalt paving operations (Miller, Hartmann \& Dorée, 2011) by analysing machine movements and changes of the surface and in-asphalt temperatures during rolling operations.

However, these previously created VR environments for rolling operations mainly concentrate on representing real or simulated scenarios and do not aim to explicate operators' tacit knowledge. As the tacit knowledge of machine operators can hardly be verbalized, we aimed at developing a tool in the form of a VR environment to assist machine operators in showing, instead of explaining, how they would proceed with the compaction process under specific circumstances. We hypothesis that the VR environment allows to explicate the operators' knowledge by collecting and studying rolling patterns in project-specific conditions that include: (1) pre-defined road geometry, such as curved roads, road junctions, and roundabouts; and (2) movements of other machines. The movements of the virtual roller, steered by an operator within the VR environment, are documented and analysed quantitatively to allow further comparing different rolling patterns.

\section{A VR APPROACH TO EXTRACT AND ANALYSE OPERATORS' TACIT KNOWLEDGE}

To develop the tool we adopted a methodology that combines ethnographic and action research principles (Hartmann, Fischer \& Haymaker, 2009). By following the methodology, we continuously discussed with paving specialists possible embodiments of site conditions by means of VR technologies, and alternative approaches to analyse rolling patterns, which are considered as operationalized compaction strategies. An iterative process of creating and testing several prototypes allowed us to rapidly develop and test the VR environment.

The developed VR environment allows machine operators to virtually construct rolling patterns in the context of a compaction project. By utilizing a clearly understandable interface and a sophisticated game engine we attempted to reduce computer literacy requirements and, at the same time, to support fluid interactions between the machine operators and the VR environment. In particular, the developed VR environment is built upon a java-based 3D game engine JMonkeyEngine 3 SDK: an open source project with a set of libraries and an integrated development environment (IDE). The engine uses a Lightweight Java Game Library (LWJGL) as a default renderer, and supports shader technology to visualize surroundings. Altogether, these factors make the game engine particularly suitable for rapid development of specialized VR environments.

During development, we aimed to reduce the level of required computer literacy. As the player's ability to "play the game" should be distinguished from level of player's knowledge in the particular domain (Virvou, Manos \& Katsionis, 2006), we specially attempted to support fluid interactions between users and the environment. For this purpose, all information is visually represented to an operator on a large whiteboard to support immersion (Bao \& Gergle, 2009) of the participants into the virtual world. The presented information include road geometry, movements of a paver and the expected cooling rate of the asphalt mixture. Only a single action - indicating the roller's destination by using a pen or a mouse - is required from the operator. The virtual roller moves according to the operator's decisions and, in this way, construct a rolling pattern as input data for further analysis. The movements of a virtual roller are documented, analysed, and visualized.

The proposed approach to explicate and analyse the operators' tacit knowledge on how to compact in particular conditions is constituted by data collection, analysis, and visualization components (Figure 2). To this end, in a first step, the parts of a rolling pattern are combined together into segments that represent movements of a roller without changing the main heading direction. Additionally, the homogeneity of coverage of the asphalt mat as the roller moves is calculated. The coverage is calculated by 
discretizing the compacted surface into triangular elements and computing how many times the roller travelled over every spot.

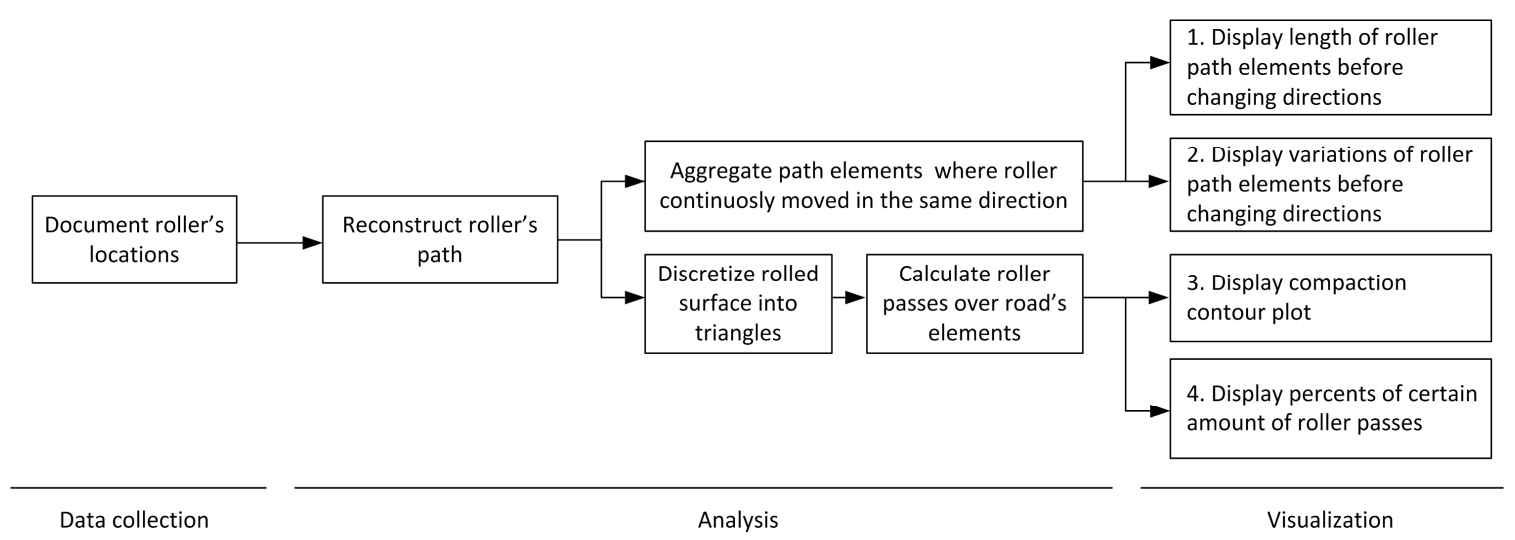

Figure 2 - An approach to analyse operationalized operators' tacit knowledge

Finally, the analysed rolling patterns are visualized as bar charts and as contour plots (Miller, Hartmann \& Dorée, 2011). In this way, the segments of the rolling patterns are represented as variations in relation to the average length of the rolling segments. The processed data and the obtained visualization can be cross-compared to identify good and bad strategies by both qualitative and quantitative analysis.

The created visualizations and the quantitative analysis of rolling patterns solely rely on the input data from the VR environment. Therefore, interactions between the VR environment and the roller operators are crucial for success of reconstructing rolling patterns, thus requiring an understandable and easy-to-use interface.

\section{Testing the Proposed Approach: Constructing and Analysing Rolling Patterns}

To evaluate the approach in general and the VR environment in particular, we enrolled two civil engineering specialists who are heavily involved in analysing asphalt compaction processes and educating roller operators. The specialists were asked to construct illustrative rolling patterns for a hypothetical compaction project that include a patch of a curved road. The illustrative implementations were suggested to represent some pitfalls in the compaction process. One of the created rolling patterns and the corresponding analysis are shown in Figures 3 and 4. The analysis of the created visualizations clearly shows variations in rolling segments in relation to their average value. Besides, a compaction contour plan demonstrates that the road was not homogeneously covered by the travelled roller: the curve by itself was undercompacted while some parts of the road received significantly more compaction than others. Quantitative analysis of the compaction contour plot shows that the roller travelled over 50\% of the road two or three times, while other parts of the road were compacted only once or more than three times.

While testing the VR environment the enrolled civil engineers indicated no difficulties during constructing rolling patterns within the VR environment and acknowledged the intuitiveness of the interface. However, they suggested including an acquaintance period for new users as a part of the developed case of the construction project. During this period machine operators could familiarize themselves with the environment and project the real-world dimensions of the machine and the dimensions within the VR environment.

The obtained visualization formats were also discussed with the enrolled engineers who found them as adequate descriptions of rolling patterns. Altogether, the proposed method was considered as an adequate means to explicate operators' tacit knowledge on how to proceed with the compaction process. 


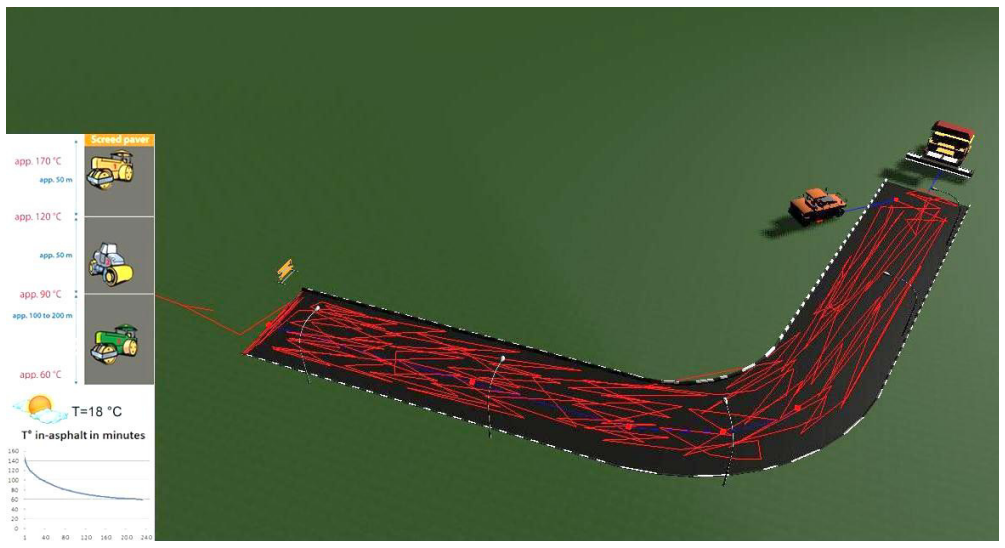

Figure 3 - An exemplary rolling pattern constructed within the VR environment
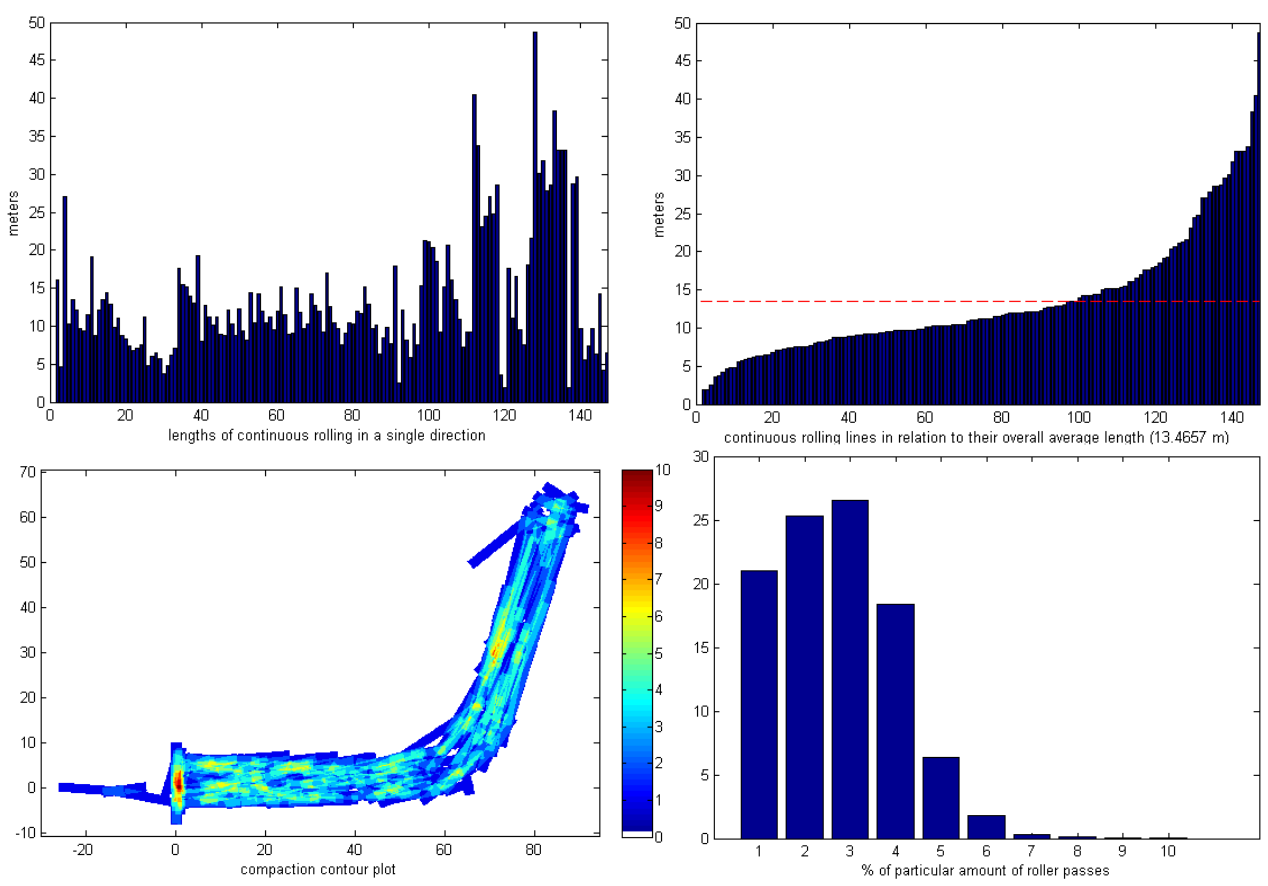

Figure 4 - Analysing the exemplary rolling pattern

\section{CONCLUSIONS AND FUTURE WORK}

Knowledge of roller operators is tacit and, therefore, is hard to be verbalized directly. Thus, before disseminating, the operators' knowledge need to be explicated. This paper describes an approach on how to extract operators' knowledge as decisions on how to process with the compaction task in particular conditions. The machine movements are documented within the specially developed VR environment and, later, analyzed. Potentially, the recorded rolling patterns can be compared with the operators' intended compaction strategy, described by an operator before constructing the rolling pattern. The acquired quantitative description of a particular rolling pattern allows comparison of different patterns.

The future research on using VR environments with more than one operator can provide additional insight into how machine operators do collaborate during the compaction process. We see the 
development and utilization of a multi-user serious gaming environment as a promising approach in this case. Ultimately, the better understanding of the operators' compaction strategies can be seen as a stepping stone in alignment and integration of such strategies into automation and robotization of compaction process.

\section{ACKNOWLEDGEMENTS}

The authors would like to acknowledge Frank Bijleveld and Wytse Mensonides, members of the ASPARi (Asphalt Paving and Innovation) network, for fruitful discussions. This research is supported by the Pioneering Foundation, province of Overijssel, the Netherlands.

\section{REFERENCES}

Asphalt-Institute (1989) The Asphalt Handbook, ed. manual series No.4.

Bao, P., \& Gergle, D. (2009) What's “this” you say? The use of local references on distant displays, Proceedings of the 27th International Conference on Human Factors in Computing Systems, Boston, MA, USA.

Chavada, R., \& Dawood, N.N. (2010) An innovative approach to integrate H\&S issues in the construction project planning using serious game engine technologies, Proceedings of the 10th international conference on construction applications of virtual reality, Sendai, Japan.

Cheng, T., \& Teizer, J. (2012) Real-time resource location data collection and visualization technology for construction safety and activity monitoring applications, Automation in Construction.

Floss, R. (2001) Compaction Technology in Earthwork, Highway and Transportation Engineering, BOMAG Fachbuch, Boppard.

Greenfield, P.M. (2009) Technology and informal education: what is taught, what is learned, Science, 323, 69-71.

Hartmann, T., Fischer, M., \& Haymaker, J. (2009) Implementing information systems with project teams using ethnographic-action research. Advanced Engineering Informatics, 23, 57- 67.

Huerne ter, H., Miller, S., \& and Dorée, A.G. (2007) New technologies in the paving process need to be based on "common practice" and operators' heuristics. International Symposium on Maintenance and Rehabilitation of Pavements and Technological Control, Mairepav'05, 8-10 August, Park City, Utah, USA.

Kang, L.S., Moon, H.S., Dawood, N., \& Kang, M.S. (2010) Development of methodology and virtual system for optimized simulation of road design data, Automation in Construction, 19(8), 10001015.

Krogh von, G., Ichijo, K., \& Nonaka, I. (2000) Enabling Knowledge Creation: How to Unlock the Mystery of Tacit Knowledge and Release the Power of Innovation, Oxford University Press, USA.

Lavin, A. (2003) Asphalt Pavements: A Practical Guide to Design, Production and Maintenance for Engineers and Architects, Taylor \& Francis.

Mikropoulos, T.A. \& Natsis, A. (2011) Educational virtual environments: A ten-year review of empirical research (1999-2009). Computers \& Education, 56(3), 769-780. 
Miller, S.R. (2010). Hot mix asphalt construction, towards a more professional approach (Doctoral dissertation). University of Twente, Enschede, The Netherlands.

Miller, S.R., Hartmann, T., \& Dorée, A.G. (2011) Measuring and visualizing hot mix asphalt concrete paving operations. Automation in Construction, 20(4), 474-81.

NAPA (1996) Rolling and compaction of Hot Mix Asphalt Pavement, National Asphalt Pavement Association, Information series, 1996.

NCDoTMaT (2012) Superpave. Hot Mix Asphalt Quality Management System. Retrieved from North Carolina Department of Transportation website: https:/connect.ncdot.gov/resources/Materials/ Materials/2012\%20QMS\%20Asphalt\%20Manual.pdf.

Nonaka, I., \& Takeuchi, H. (1995) The knowledge-creating company : how Japanese companies create the dynamics of innovation. New York: Oxford University Press.

Retik, A. \& Shapira, A. (1999) VR-based planning of construction site activities, Automation in Construction, vol. 8, no. 6, pp. 671-680.

Tserng, H.-P., Russell, J.S., Krishnamurth, B.K., \& Schmitt, R.L. (1996) An operations planning system for asphalt pavement compaction, Proceedings of the 13th ISARC (pp.349-358), Tokyo, Japan.

Turkiyyah, G.M., \& Northwest, T. (2007) SIMPAVE: towards building interactive simulations for planning pavement construction: Transportation Northwest.

Vasenev, A., T. Hartmann \& Dorée, A. G. (2012) Prediction of the in-asphalt temperature for road construction operations, ASCE International Conference on Computing in Civil Engineering, Clearwater Beach, FL, USA.

Virvou, M., Manos, K., \& Katsionis, G. (2006) Virtual Reality Edutainment: cost-effective development of personalised software applications. Computational Science and Its Applications, 3980, 251-60. 